Research Article

\title{
Mobile Interface CMS Method for Vibration Characteristics Prediction of Mistuned Bladed Disk with Different Coupling Degrees
}

\author{
Liang Zhang $\mathbb{D}^{\mathrm{D}}$, Qidi Wang, and Xin Li \\ Faculty of Mechanical Engineering and Automation, Liaoning University of Technology, Jinzhou, China \\ Correspondence should be addressed to Liang Zhang; zhangliang545238@163.com
}

Received 5 October 2021; Revised 13 November 2021; Accepted 9 December 2021; Published 29 December 2021

Academic Editor: Jaroslaw Latalski

Copyright (C) 2021 Liang Zhang et al. This is an open access article distributed under the Creative Commons Attribution License, which permits unrestricted use, distribution, and reproduction in any medium, provided the original work is properly cited.

\begin{abstract}
The prediction of vibration characteristics was studied in the mistuned bladed disk by the mobile interface prestressed component mode synthesis (CMS) superelement method. When the strongly, generally, and weakly coupling in the mistuned bladed disk, according to the results of the direct FEM method, the prediction accuracy of this method was verified and compared with the fixed-interface CMS method by using the relative error of dynamic frequency, vibration mode matching function, and dimensionless root mean square error of vibration amplitudes. It is pointed that for mistuned bladed disk in the strong coupling, the prediction accuracy of dynamic frequency and vibration amplitudes are higher by the mobile interface CMS method and the vibration modes are matched with the direct method. In weak coupling, the results of dynamic frequency and vibration modes predicted by the mobile interface CMS method and the fixed-interface CMS method are consistent with the direct method, but the vibration amplitudes' prediction error of the mobile interface CMS method is lower than that of the fixed-interface CMS method. In general coupling, the mobile interface CMS method has higher dynamic frequency prediction accuracy at low order, and the two methods have comparable dynamic frequency prediction accuracy at high order. The vibration modes predicted by the two methods are matched with the direct FEM method, and the prediction accuracy of vibration amplitude by the mobile interface CMS method is better than that of the fixed-interface CMS method. The results indicate that the mobile interface CMS method could more accurately predict vibration characteristics of the mistuned bladed disk with different coupling degrees and could be an effective measurement for studying the vibration characteristics of the mistuned bladed disk system.
\end{abstract}

\section{Introduction}

The bladed disk is an extremely important component in a gas turbine engine, and its vibration characteristics are directly related to the performance and safety of the engine. In the design stage, the finite element method is mainly used to predict the vibration characteristics of the bladed disk. Theoretically, the bladed disk is a harmonic structure; that is, it satisfies the condition of cyclic symmetry. Only the finite element model of the basic sector of the bladed disk is established, and the cyclic symmetry method can be used to predict its vibration characteristics. Literature [1] predicts the vibration characteristics of a gas turbine with a crowned harmonic blisk on the basis of the cyclic symmetry method. In practice, due to the influence of factors such as mechanical manufacturing error, material parameter dispersion, wear in use, and chatter suppression, there are slight differences between the blades, which are mistuned [2]. The periodic mistuned disk still satisfies the condition of cyclic symmetry, and the cyclic symmetry method can be used to predict its vibration characteristics. Literature [3] analyzed the vibration localization of the periodic offset mistuned bladed disk using the cyclic symmetry method. However, the cyclic symmetry method will fail when any mistuned disk destroys the cyclic symmetry condition of the laded disk structure. The overall model of the bladed disk must be established, and the direct method must be used to predict its vibration characteristics. Literature [4] calculated the dynamic frequency of a mistuned bladed disk under rated conditions on the basis of the direct method. Lee [5] 
analyzed the forced vibration characteristics of the mistuned bladed disk using the direct method. Kan [6] investigated the effect of Coriolis force on the forced response of the mistuned bladed disk using the direct method. When the size of the bladed disk is large and the twisted shape of the blade is complex, in order to ensure the prediction accuracy, the number of units and nodes divided into the finite element model of the whole bladed disk is large, which makes the direct method very time-consuming and even impossible to complete the prediction. Therefore, it is necessary to adopt an efficient reduction method to predict the vibration characteristics of the mistuned bladed disk structure [7], which not only greatly reduces the number of degrees of freedom of the model but also satisfies the prediction accuracy requirements of the vibration characteristics.

The order reduction methods mainly include the static reduction method $[8,9]$, dynamic reduction method [10], and modal synthesis method. Modal synthesis methods include the fixed-interface method [11], free-interface method [12, 13], and modal synthesis superelement method [14]. Literature [15] developed and verified the component modal mistuned method. Literature [16] analyzed the static frequency of a gas turbine's mistuned bladed disk structure based on the modal synthesis superelement method. The papers $[17,18]$, respectively, proposed a reduction analysis method of the dynamic characteristics of the mistuned bladed disk, but there were not consider the influence of the centrifugal force of the rotational speed. The papers $[19,20]$ proposed a fixed-interface CMS method that combines a fixed-interface prestress and a free-interface CMS superelement method. Literature [21-25] carried out related researches on the vibration characteristics of mistuned bladed disks on the basis of the fixed-interface CMS method. Literature [26] identified the mistuned parameters of real blades using modal testing and finite element method, and the vibration characteristics of the real mistuned bladed disk structure were analyzed by the mobile interface prestressed CMS superelement method.

At present, the vibration characteristics of the mistuned bladed disk are mainly used in the fixed-interface CMS method. When the rotational speed prestresses analysis, an approximately fixed displacement constraint is applied to the base sector coupling surface of the bladed disk, which approximates the prediction accuracy of the vibration characteristics of the mistuned bladed disk. Based on the mobile interface prestress CMS superelement method that predicts the vibration characteristics of different coupling mistuned bladed disk structures, using the relative error of dynamic frequency, vibration mode matching function and dimensionless root mean square error of vibration amplitudes verify the prediction accuracy of the method, compared with the fixed-interface CMS method.

\section{Establishment of Mistuned Blade Disk Model with Different Coupling Degrees}

The coupling degree of the bladed disk is the characterization of the rigid relationship between the blade and the disk. When the rigidity of the blade and the disk is close, the vibration of the blade can cause strong vibration of the disk, which is a strong coupling bladed disk. When the blade rigidity is not very small relative to the wheel disk, and the vibration of the blade and the wheel disk affect each other greatly, it is a general coupling bladed disk. When the blade rigidity is much smaller than the wheel disk, the vibration of the blade can only cause the weak vibration of the wheel disk, which is a weakly coupled bladed disk. In this paper, the basic sector and bladed disk system finite element models of strong coupling, weak coupling, and general coupling are established as shown in Figures 1(a)-1(c).

The bladed disk working speed is $11353 \mathrm{r} / \mathrm{min}$. The number of blades of the strong coupled bladed disk is 12 . The density of the bladed disk material is $4500 \mathrm{~kg} / \mathrm{m}^{3}$, and Poisson's ratio is 0.3 . The harmonic modulus of elasticity is $2.1 \times 10^{11} \mathrm{~Pa}$. The number of blades of the generally coupled bladed disk is 38 , the elastic modulus of the disk material is $1.15 \times 10^{11} \mathrm{~Pa}$, Poisson's ratio is 0.3 , and the density is $4640 \mathrm{~kg} / \mathrm{m}^{3}$. The density of the blade material is $4380 \mathrm{~kg} / \mathrm{m}^{3}$, Poisson's ratio is 0.3 , and the harmonic modulus of elasticity is $1.135 \times 10^{11} \mathrm{~Pa}$. The number of blades of the weakly coupled bladed disk is 8 , Poisson's ratio of the bladed disk material is 0.3 , the density is $7800 \mathrm{~kg} / \mathrm{m}^{3}$, and the harmonic modulus of elasticity is $2.1 \times 10^{11} \mathrm{~Pa}$. Using different simulation mistuned disks of the blade elastic modulus, Figures 2(a)-2(c) show the mistuned elastic modulus of each blade for three coupling degrees.

\section{Mobile Interface CMS Method}

In this paper, the vibration characteristics of the mistuned blisk system are predicted using the mobile interface prestressed CMS superelement method. The mobile interface CMS method consists of two parts: the mobile interface prestressed analysis and the free-interface CMS superelement analysis.

3.1. Prestressed Analysis of Mobile Interface. First, the bladed disk structure is assumed to be a harmonic system. Let the established bladed disk basic sector finite element model be the first bladed disk basic sector, and $\left\{v^{1}\right\}$ is the displacement vector of the first bladed disk basic sector. The displacement vector is divided into interface degrees of freedom $\left\{v_{m}^{1}\right\}$ and noninterface degrees of freedom $\left\{v_{s}^{1}\right\}$; there are

$$
\left\{v^{1}\right\}=\left\{\begin{array}{c}
v_{m}^{1} \\
v_{s}^{1}
\end{array}\right\} .
$$

The constraint boundary, working speed load, and interface cyclic symmetry conditions are applied, and the displacement vector $\left\{v_{m}^{1}\right\}$ of the first basic sector interface degrees of freedom of the bladed disk system at the working speed is obtained using the cyclic symmetry analysis method.

The displacement vector of the $i$-th basic sector interface degree of freedom in the blade disk system is $\left\{v_{m}^{i}\right\}$, which can be expressed in the following form: 


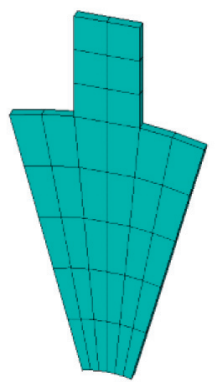

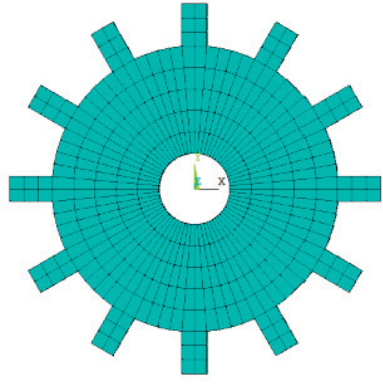

(a)
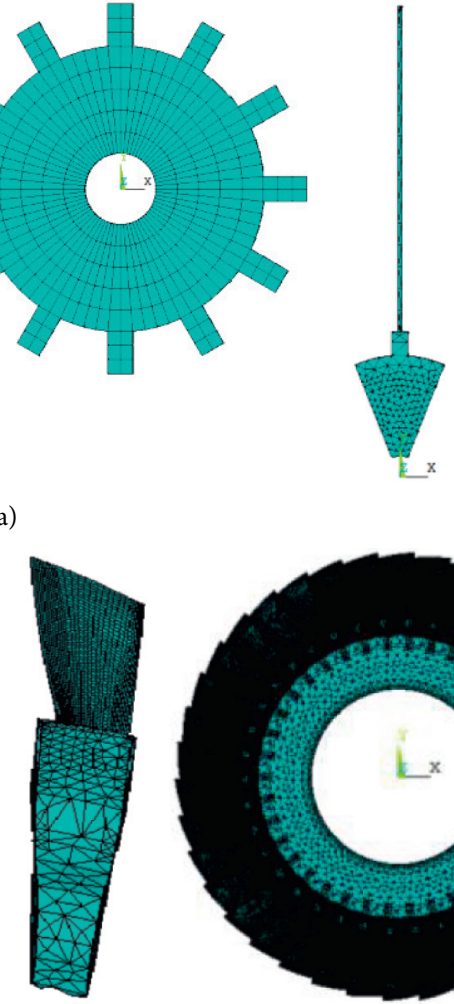

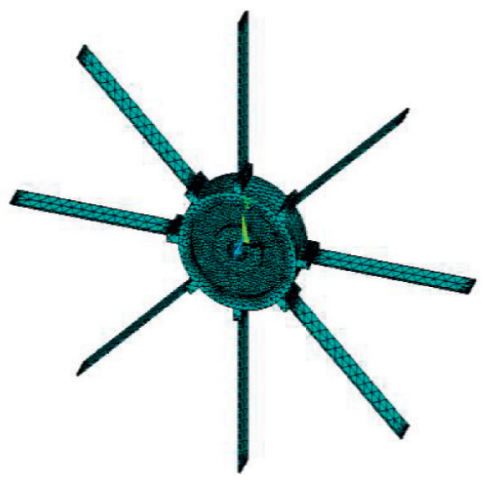

(b)

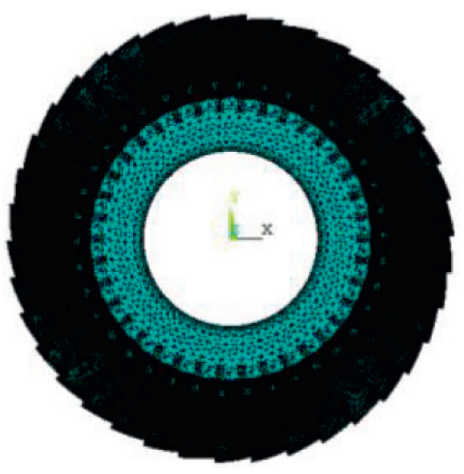

(c)

Figure 1: Finite element model of basic sector and bladed disk system with different coupling degrees. (a) Strong coupling. (b) Weak coupling. (c) General coupling.

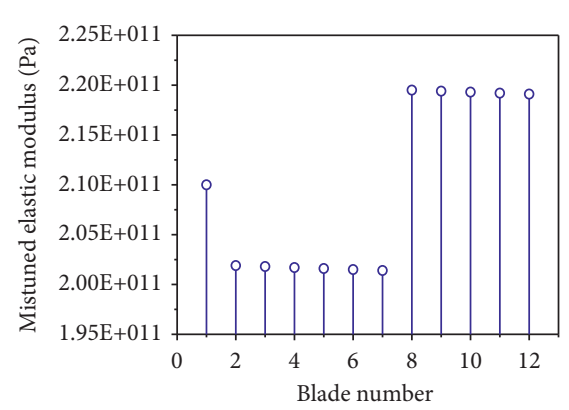

(a)

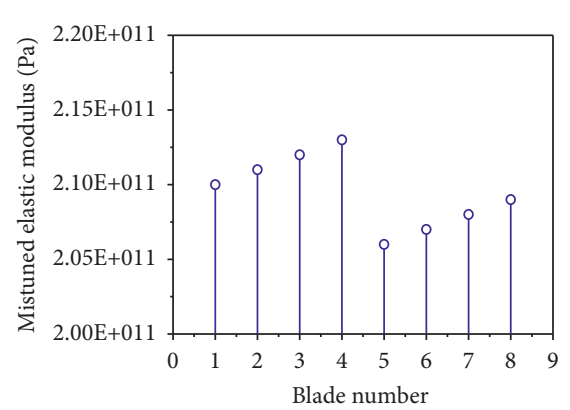

(b)

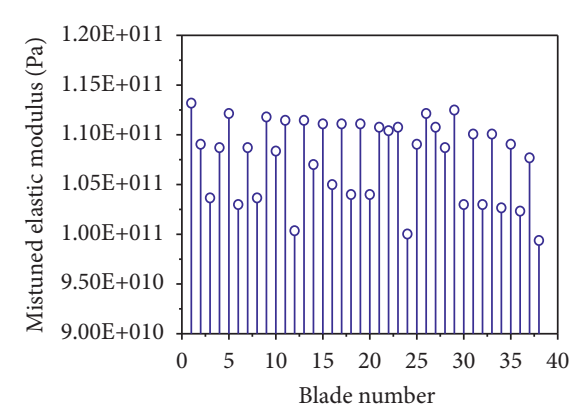

(c)

Figure 2: Mistuned elastic modulus of blades of the bladed disk with different coupling degrees. (a) Strong coupling. (b) Weak coupling. (c) General coupling.

$$
\left\{v_{m}^{i}\right\}=\left\{\begin{array}{lll}
v_{m}^{i 1} & \cdots & v_{m}^{i k}
\end{array}\right\}^{T}
$$

where $k$ represents the number of nodes on the interface; the displacement vector of the $j$-th node on the interface is

$$
\left\{v_{m}^{i j}\right\}=\left\{\Delta x_{m}^{i j} \Delta y_{m}^{i j} \Delta z_{m}^{i j}\right\}, \quad 1 \leq j \leq k .
$$

According to the cyclic symmetry condition, the displacement vector $\left\{v_{m}^{(i+1) j}\right\}$ of the $j$-th node on the interface of the $i+1$-th basic sector in the bladed disk system can be calculated using the following formula:

$$
\left\{v_{m}^{(i+1) j}\right\}^{T}=\left[\begin{array}{ccc}
\cos \left( \pm \frac{360}{N}\right) & \sin \left( \pm \frac{360}{N}\right) & 0 \\
-\sin \left( \pm \frac{360}{N}\right) & \cos \left( \pm \frac{360}{N}\right) & 0 \\
0 & 0 & 1
\end{array}\right]\left\{v_{m}^{i j}\right\}^{T}
$$

Here, $N$ is the number of blades, and the displacement vector of the interface degree of freedom of the $i+1$-th basic sector in the bladed disk system is $\left\{v_{m}^{i+1}\right\}$, which can be calculated using the following form: 


$$
\left\{v_{m}^{i+1}\right\}=\left\{\begin{array}{lll}
v_{m}^{(i+1) 1} & \cdots & v_{m}^{(i+1) k}
\end{array}\right\}^{T}
$$

Since $\left\{v_{m}^{1}\right\}$ is known, we substitute it into equations (2)-(5) to get $\left\{v_{m}^{i}\right\}, 2 \leq i \leq N$.

Secondly, the bladed disk structure is regarded as a mistuned system. The overall bladed disk is composed of $N$ substructures, and each substructure is a basic sector. At a certain speed, the static equation of substructure $i$ is

$$
\left[K^{i}\right]\left\{u^{i}\right\}=\left\{F^{i}\right\}
$$

where $\left[K^{i}\right],\left\{u^{i}\right\}$, and $\left\{F^{i}\right\}$ are, respectively, the stiffness matrix, displacement vector, and load vector generated by the rotational speed of substructure $i$. The displacement vector $\left\{u^{i}\right\}$ is divided into the following form:

$$
\left\{u^{i}\right\}=\left\{\begin{array}{c}
u_{m}^{i} \\
u_{s}^{i}
\end{array}\right\}
$$

Here, $\left\{u_{m}^{i}\right\}$ and $\left\{u_{s}^{i}\right\}$ are interface and noninterface degrees of freedom, respectively. Substitute equations (7) into (6) to get

$$
\left[\begin{array}{cc}
K_{m m}^{i} & K_{m s}^{i} \\
K_{s m}^{i} & K_{s s}^{i}
\end{array}\right]\left\{\begin{array}{c}
u_{m}^{i} \\
u_{s}^{i}
\end{array}\right\}=\left\{\begin{array}{c}
F_{m}^{i} \\
F_{s}^{i}
\end{array}\right\} .
$$

The mobile displacement constraint is applied to the degrees of freedom of the interface, i.e., $\left\{u_{m}^{i}\right\}=\left\{v_{m}^{i}\right\}$, and the prestressed analysis is carried out on the substructure. Equation (8) is simplified as

$$
\left[K_{s s}^{i}\right]\left\{u_{s}^{i}\right\}=\left\{F_{s}^{i}\right\}-\left[K_{s m}^{i}\right]\left\{v_{m}^{i}\right\} .
$$

Solve equation (9), and get the stiffness matrix $\left[K_{l}^{i}(\Omega)\right]$ and softening matrix $\left[K_{r}^{i}(\Omega)\right]$ related to the speed $\Omega$. When the effects of stiffness and softening are considered at the same time, the stiffness matrix of substructure $i$ is as follows:

$$
\left[K_{*}^{i}\right]=\left[K^{i}\right]+\left[K_{l}^{i}(\Omega)\right]-\left[K_{r}^{i}(\Omega)\right]
$$

3.2. Free-Interface CMS Superelement Analysis. For an undamped structure, the dynamic equation of substructure $i$ is

$$
\left[M^{i}\right]\left\{\ddot{u}^{i}\right\}+\left[K_{*}^{i}\right]\left\{u^{i}\right\}=\left\{\begin{array}{c}
F_{m}^{i} \\
F_{s}^{i}
\end{array}\right\}
$$

where $\left[M^{i}\right]$ is the mass matrix of the substructure, and $\left\{F_{m}^{i}\right\}$ and $\left\{F_{s}^{i}\right\}$ are the force vectors of the interface nodes of substructure $i$ on other substructures and the external force vectors of noninterface nodes, respectively.

Substituting equations (7) into (11), when the structure vibrates freely, the force exerted on noninterface nodes is $\left\{F_{s}^{i}\right\}=\{0\}$, and equation (11) is simplified as

$$
\left[\begin{array}{cc}
M_{m m}^{i} & M_{m s}^{i} \\
M_{s m}^{i} & M_{s s}^{i}
\end{array}\right]\left\{\begin{array}{c}
\ddot{u}_{m}^{i} \\
\ddot{u}_{s}^{i}
\end{array}\right\}+\left[\begin{array}{cc}
K_{* m m}^{i} & K_{* m s}^{i} \\
K_{* s m}^{i} & K_{* s s}^{i}
\end{array}\right]\left\{\begin{array}{c}
u_{m}^{i} \\
u_{s}^{i}
\end{array}\right\}=\left\{\begin{array}{c}
F_{m}^{i} \\
0
\end{array}\right\} .
$$

The mobile displacement constraints of the interface degrees of freedom are analyzed and released using the freeinterface CMS superelement method, that is, delete the constraint $\left\{u_{m}^{i}\right\}=\left\{v_{m}^{i}\right\}$, and the undamped free vibration equation of the substructure $i$ with the unconstrained interface is

$$
\left[M^{i}\right]\left\{\ddot{u}^{i}\right\}+\left[K_{*}^{i}\right]\left\{u^{i}\right\}=\{0\} .
$$

From equation (13), modal truncation is used to obtain the low-order modal set and the modal coordinate transformation matrix corresponding to the free-interface method. Taking the modal coordinate transformation matrix as the approximate modal of substructure $i$, substituting the modal coordinate transformation formula into equation (12), the dynamic equation of substructure $i$ in modal coordinates is obtained as

$$
\left[\widehat{M}^{i}\right]\left\{\ddot{q}_{D}^{i}\right\}+\left[\widehat{K}_{*}^{i}\right]\left\{q_{D}^{i}\right\}=\left\{\widehat{F}^{i}\right\}, \quad i=1,2, \ldots, N .
$$

Here, $\left\{q_{D}^{i}\right\}$ is the modal coordinate vector, $\left[\widehat{M}^{i}\right]=\left[T_{D}^{i}\right]^{T}\left[M^{i}\right]\left[T_{D}^{i}\right]$ is the mass matrix under the modal coordinate, $\left[\widehat{K}_{*}^{i}\right]=\left[T_{D}^{i}\right]^{T}\left[K_{*}^{i}\right]\left[T_{D}^{i}\right]$ is the stiffness matrix under the modal coordinate, $\left\{\widehat{F}^{i}\right\}=\left[T_{D}^{i}\right]^{T}\left\{\begin{array}{c}F_{m}^{i} \\ 0\end{array}\right\}$ is the load vector, and $\left[T_{D}^{i}\right]$ is the modal coordinate transformation matrix.

Combining the dynamic equations of $N$ substructures forms the reduced dynamic equation of the overall mistuned bladed disk structure in modal coordinates:

$$
\left[\begin{array}{ccc}
{\left[\widehat{M}^{1}\right]} & 0 & 0 \\
0 & \ddots & 0 \\
0 & 0 & {\left[\widehat{M}^{N}\right]}
\end{array}\right]\left\{\begin{array}{c}
\ddot{q}_{D}^{1} \\
\vdots \\
\ddot{q}_{D}^{N}
\end{array}\right\}+\left[\begin{array}{ccc}
{\left[\widehat{K}_{*}^{1}\right]} & 0 & 0 \\
0 & \ddots & 0 \\
0 & 0 & {\left[\widehat{K}_{*}^{N}\right]}
\end{array}\right]\left\{\begin{array}{c}
q_{D}^{1} \\
\vdots \\
q_{D}^{N}
\end{array}\right\}=\left[\begin{array}{ccc}
{\left[T_{D m}^{1}\right]^{T}} & 0 & 0 \\
0 & \ddots & 0 \\
0 & 0 & {\left[T_{D m}^{N}\right]^{T}}
\end{array}\right]\left\{\begin{array}{c}
F_{m}^{1} \\
\vdots \\
F_{m}^{N}
\end{array}\right\} .
$$

The displacement coordination and force balance conditions are adopted to couple the degree of freedom of interface nodes of the substructure to obtain the generalized coordinate transformation matrix. The generalized coordinate transformation formula is substituted into equation (15) to obtain the dynamic equation of the overall mistuned bladed disk structure under the generalized coordinates including the effects of rigidization and softening: 


$$
\begin{aligned}
& {[\bar{M}]\{\ddot{p}\}+\left[\bar{K}_{*}\right]\{p\}=\{\bar{F}\},} \\
& {[\bar{M}]=[a]^{T}\left[\begin{array}{ccc}
{\left[\hat{M}^{1}\right]} & 0 & 0 \\
0 & \ddots & 0 \\
0 & 0 & {\left[\widehat{M}^{N}\right]}
\end{array}\right][a],} \\
& {\left[\bar{K}_{*}\right]=[a]^{T}\left[\begin{array}{ccc}
{\left[\widehat{K}_{*}^{1}\right]} & 0 & 0 \\
0 & \ddots & 0 \\
0 & 0 & {\left[\widehat{K}_{*}^{N}\right.}
\end{array}\right][a],} \\
& \{\bar{F}\}=[a]^{T}\left[\begin{array}{ccc}
{\left[T_{D m}^{1}\right]^{T}} & 0 & 0 \\
0 & \ddots & 0 \\
0 & 0 & {\left[T_{D m}^{N}\right.}
\end{array}\right]\left\{\begin{array}{c}
F_{m}^{1} \\
\vdots \\
F_{m}^{N}
\end{array}\right\},
\end{aligned}
$$

where $\{p\}$ is the reduced generalized coordinate vector, and $[a]$ is the generalized coordinate transformation matrix.

The free vibration equation for the reduction of the overall mistuned bladed disk is

$$
[\bar{M}]\{\ddot{p}\}+\left[\bar{K}_{*}\right]\{p\}=\{0\} .
$$

By solving equations (16) and (17), the dynamic frequency, the response, and mode shape under the reduced generalized coordinate $\{p\}$ of the overall mistuned bladed disk can be obtained, and the superelement can be used.

The generalized coordinate inverse transformation and the modal coordinate inverse transformation are used to obtain the response and mode shape of the overall mistuned bladed disk under the physical coordinate $\{u\}$ and complete the extended superelement.

\section{Influence of Different Coupling Degrees on Prediction Accuracy of Vibration Characteristics}

The vibration characteristics of the mistuned bladed disk system mainly include the vibration frequency, vibration shape, and amplitude. In this paper, the prediction result of the direct method is used as the theoretical value, and the relative error of dynamic frequency, vibration mode matching function, and dimensionless root mean square error of vibration amplitudes are introduced to verify the prediction accuracy of the mobile interface CMS method and compare it with the fixed-interface CMS method.

\subsection{Prediction Accuracy Index}

4.1.1. Relative Error of Dynamic Frequency. The dynamic frequency relative error prediction of the bladed disk system at a certain speed is

$$
e_{d d f r e}^{l}=\left|\frac{F_{c s}^{l}-F_{m f c m s}^{l}}{F_{c s}^{l}}\right| \times 100 \% .
$$

Here, $l$ is the mode order, $F_{c s}^{l}$ is the $l$ th-order dynamic frequency of the mistuned bladed disk system obtained by the direct method, and $F_{m f_{c m s}}^{l}$ is the $l$ th-order dynamic frequency obtained by the mobile interface CMS superelement method or the fixed-interface CMS superelement method.

4.1.2. Vibration Mode Matching Function. The dimensionless amplitude vector of the $l$-th mode shape of the mistuned bladed disk predicted by the direct method is

$$
\left\{X_{l z}\right\}=\left\{x_{l z}^{1}, x_{l z}^{2}, \ldots, x_{l z}^{k}, \ldots, x_{l z}^{N}\right\} .
$$

The dimensionless amplitude vector of the $l$-th mode shape of the mistuned bladed disk predicted by mobile interface CMS method or fixed-interface CMS method is

$$
\left\{X_{l}\right\}=\left\{x_{l}^{1}, x_{l}^{2}, \ldots, x_{l}^{k}, \ldots, x_{l}^{N}\right\} .
$$

Taking the dimensionless amplitude vector element sequence predicted by the direct method as the template sequence, the matching degree function between sequence $\left\{X_{l}\right\}$ and template sequence $\left\{X_{l z}\right\}$ is as follows:

$$
C_{l}(\tau)=\sum_{k=1}^{N} x_{l}^{k+\tau} x_{l z}^{k}, \quad \tau=0,1,2, \ldots, N-1 .
$$

When $N<k+\tau<2 N, x_{l}^{k+\tau}=x_{l}^{k+\tau-N}$.

The parameter $\tau$ is the independent variable of the mode matching degree function $C_{l}(\tau)$, assuming that the maximum value of $C_{l}(\tau)$ is $C_{l}\left(\tau_{m}\right)$, that is,

$$
\max C_{l}(\tau)=C_{l}\left(\tau_{m}\right) .
$$

When $\tau_{m}=0$, the mode shapes predicted by the mobile interface CMS method or the fixed-interface CMS method match those of the direct method. When $\tau_{m} \neq 0$, the mode shapes do not match.

4.1.3. Dimensionless Root Mean Square Error of Vibration Amplitudes. The dimensionless root mean square error of the vibration amplitude vector element of the $l$-th mode shape of the bladed disk structure at a certain speed is

$$
\operatorname{RMSE}_{l}=\sqrt{\frac{1}{N} \sum_{k=1}^{N}\left(x_{l}^{k}-x_{l z}^{k}\right)^{2}},
$$

where $N$ is the number of blades, $x_{l}^{k}$ is the dimensionless amplitude value of the $k$-th blade in the $l$-th mode obtained by mobile interface method or fixed-interface method, and $x_{l z}^{k}$ is the dimensionless amplitude value of the $k$-th blade in the $l$-th mode obtained by the direct method.

\subsection{Prediction Accuracy Verification and Comparative Analysis}

4.2.1. Dynamic Frequency Relative Error Analysis. The dynamic frequency relative error of the strongly coupled mistuned blisk system obtained by the mobile interface CMS superelement method and the fixed-interface CMS superelement method is shown in Figure 3. From Figure 3, it can be seen that the dynamic frequency maximum relative error predicted by the fixed-interface method is $29.814 \%$. As the 


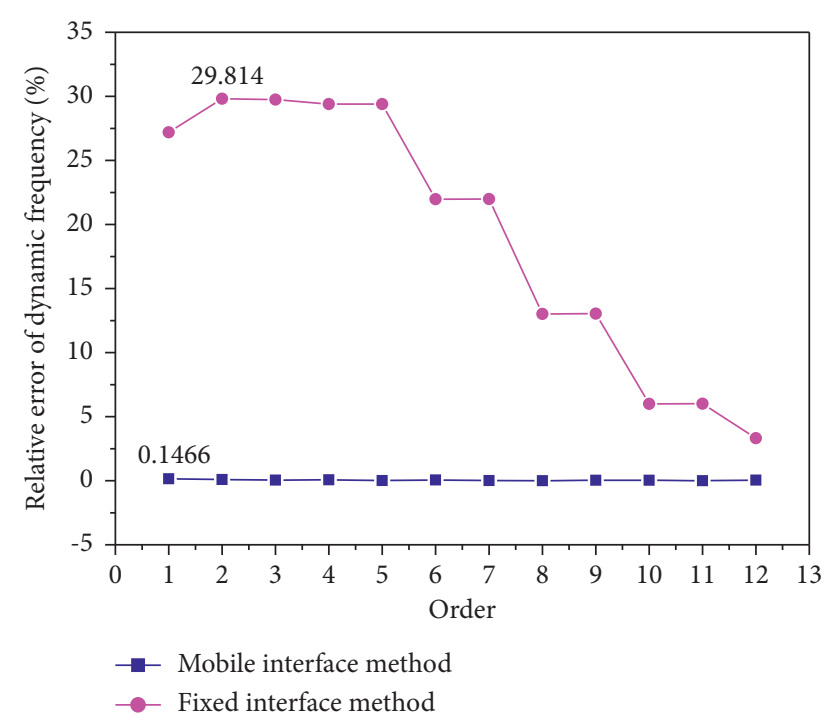

FIGURE 3: First-order bending dynamic frequency relative error of strongly coupling mistuned bladed disk.

order increases, the relative error shows a tendency to become smaller. The dynamic frequency maximum relative error predicted by the mobile interface method is $0.1466 \%$. As the order increases, the relative error does not change significantly. For the strongly coupled mistuned bladed disk structure, the mobile interface method has higher accuracy in predicting the dynamic frequency, while the fixed-interface method has lower accuracy.

Table 1 shows the dynamic frequency of the weakly coupled mistuned bladed disk system predicted by the direct method, the mobile interface method, and the fixed-interface method at a certain speed. It can be seen from Table 1 that the dynamic frequency prediction results of the mobile interface method and the fixed-interface method are consistent with the direct method, and the dynamic frequency relative error is $0 \%$. For the weakly coupled mistuned bladed disk system, both the mobile interface method and the fixed-interface method have higher dynamic frequency prediction accuracy.

Figure 4 shows the dynamic frequency relative error of the general coupling mistuned bladed disk structures obtained by the direct method, the mobile interface method, and the fixed-interface method. As can be seen from Figure 4, the dynamic frequency relative error of the general coupled mistuned bladed disk structure predicted by the mobile interface method is small, and it does not change significantly with the increase of order. However, the relative error of the general coupled mistuned bladed disk structure obtained by the fixed-interface method is larger at low order, especially when the order is no more than 5, and tends to decrease with the increase of the order. However, the relative error of the coupled mistuned bladed disk structure is smaller at high order. For general coupled mistuned bladed disk systems, the prediction accuracy of the mobile interface method is much better than that of the fixed-interface method when the order is lower, and the prediction accuracy of the two methods is similar when the order is higher.
4.2.2. Vibration Shape Matching Analysis. The mode shape matching function and parameters of the strongly coupled mistuned bladed disk predicted by the mobile interface method and the fixed-interface method are shown in Table 2. As can be seen from Table 2, when the mobile interface method is used to predict the 12th order mode of a bending family, when the mode matching degree parameter $\tau_{m}=0$, the mode matching degree function $C_{l}(\tau)$ takes the maximum value, indicating that the mode shapes of the strongly coupled mistuned bladed disk predicted by the mobile interface method match the mode shapes of the direct method. When the fixed-interface method is used to predict the mode shape of the 5 th order, when $\tau_{m}=6$, the mode matching degree function $C_{l}(\tau)$ takes the maximum value. In the 10th and 11 th modes, when $\tau_{m}=1, C_{l}(\tau)$ takes the maximum. It is shown that the mode shapes of the 5th, 10th, and 11th order of the strongly coupled mistuned bladed disk predicted by the fixed-interface method do not match those predicted by the direct method.

Figures 5(a) and 5(b) are the vibration shape matching function of the weakly coupled mistuned bladed disk predicted by the mobile interface method and the fixed-interface method, respectively. It can be seen from Figures 5(a) and 5 (b) that when the mobile interface method and the fixed-interface method are used, respectively, for a bending group of 8 th order mode, when the mode matching degree parameter $\tau_{m}=0$, the mode matching degree function $C_{l}(\tau)$ takes the maximum value. For the weakly coupled mistuned bladed disk structure, the mode shapes predicted by the mobile interface method and the fixed-interface method are matched with those predicted by the direct method.

The mode matching degree functions of the general coupled mistuned bladed disk predicted by the mobile interface method and the fixed-interface method are shown in Figures 6(a) and 6(b), respectively. It can be seen from Figures 6(a) and 6(b) that based on the prediction of the mobile interface method and the fixed-interface method, for the first 10 modes of a bend group, when $\tau_{m}=0, C_{l}(\tau)$ takes the maximum value. For the general coupled mistuned bladed disk structure, the mode shape predicted by the above two methods matches the mode shape predicted by the direct method.

\subsubsection{Dimensionless Vibration Amplitude Root Mean Square} Error Analysis. Figures 7-9 show the variation curves of dimensionless amplitude root mean square error of strongly coupled, weakly coupled, and generally coupled mistuned bladed disks with the order of vibration modes, respectively. As can be seen from Figures 7-9, for different coupling degrees of mistuned bladed disks, the dimensionless amplitude root mean square errors predicted by the mobile interface method are all smaller than those predicted by the fixed-interface method.

4.2.4. Comparison of Analysis Accuracy and Analysis Time. The analysis accuracy of dynamic frequencies and analysis time of the three methods are compared. Since the general coupling model has the largest total number of degrees of 
TABLE 1: Dynamic frequency of weakly coupling mistuned bladed disk at working speed.

\begin{tabular}{lccc}
\hline Order & \multicolumn{2}{c}{ Dynamic frequency $(\mathrm{Hz})$} \\
Direct method & Mobile interface method & Fixed-interface method \\
\hline 1 & 259.84 & 259.84 & 259.84 \\
2 & 259.93 & 259.93 & 259.93 \\
3 & 259.98 & 259.98 & 259.98 \\
4 & 260.03 & 260.03 & 260.03 \\
5 & 260.07 & 260.07 & 260.07 \\
6 & 260.12 & 260.12 & 260.12 \\
7 & 260.17 & 260.17 & 260.17 \\
8 & 260.22 & 260.22 & 260.22 \\
\hline
\end{tabular}

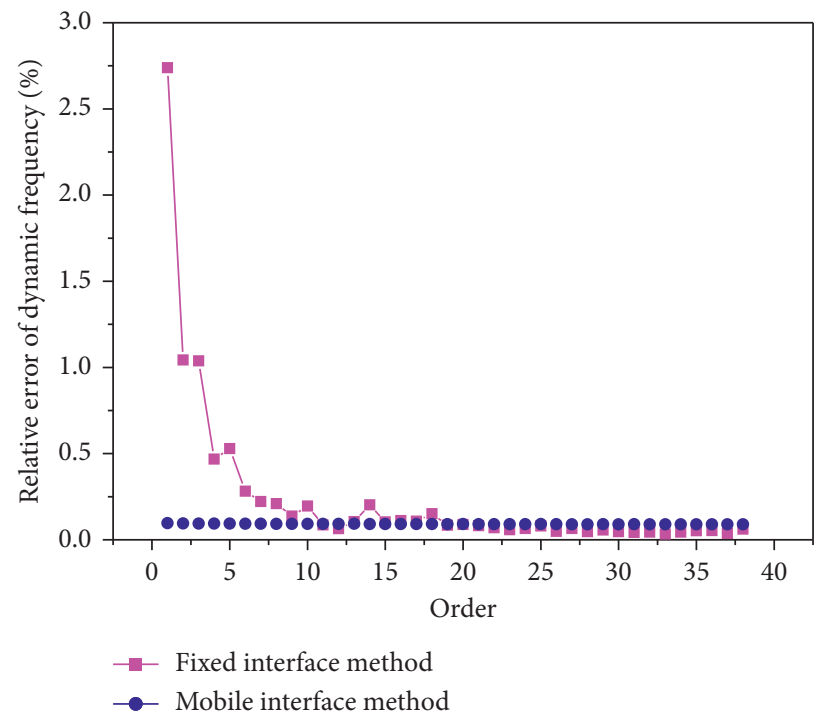

FIGURE 4: First-order bending dynamic frequency relative error of generally coupling mistuned bladed disk.

TABLE 2: Vibration mode matching degree of strongly coupling mistuned bladed disk.

\begin{tabular}{|c|c|c|c|c|}
\hline \multirow{2}{*}{ Mode shape order } & \multicolumn{2}{|c|}{ Mobile interface method } & \multicolumn{2}{|c|}{ Fixed-interface method } \\
\hline & $\max C_{l}(\tau)$ & $\tau_{m}$ & $\max C_{l}(\tau)$ & $\tau_{m}$ \\
\hline 1 & 1.5534 & 0 & 1.43309 & 0 \\
\hline 2 & 1.83892 & 0 & 1.66608 & 0 \\
\hline 3 & 1.83934 & 0 & 1.66948 & 0 \\
\hline 4 & 2.5042 & 0 & 2.20679 & 0 \\
\hline 5 & 2.61836 & 0 & 2.31116 & 6 \\
\hline 6 & 3.53852 & 0 & 3.1394 & 0 \\
\hline 7 & 3.53448 & 0 & 3.13897 & 0 \\
\hline 8 & 5.09595 & 0 & 4.67008 & 0 \\
\hline 9 & 5.16633 & 0 & 4.81133 & 0 \\
\hline 10 & 6.13409 & 0 & 5.94246 & 1 \\
\hline 11 & 6.15465 & 0 & 5.96189 & 1 \\
\hline 12 & 6.39985 & 0 & 6.25401 & 0 \\
\hline
\end{tabular}

freedom and the reduction rate of degrees of freedom, so only the analysis time of the general coupling model is compared in Table 3. As shown from Table 3, the analysis accuracy of dynamic frequencies of strong coupling bladed disk model based on the mobile interface method and the fixed-interface method is $99.8534 \%$ and $70.186 \%$, respectively; the analysis accuracy of dynamic frequencies of the general coupling bladed disk model is $99.9039 \%$ and $97.1675 \%$, respectively, and the relative analysis time is $28.42 \%$ and $27.07 \%$, respectively; the analysis accuracy of dynamic frequencies of the weak coupling bladed disk model is $100 \%$. The analysis time of the mobile interface method is 


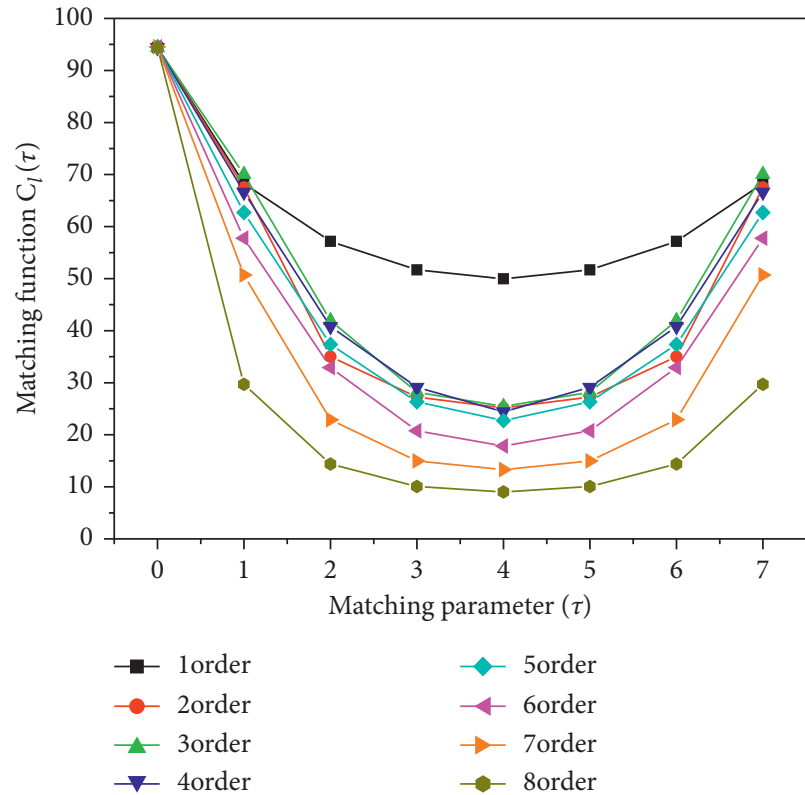

(a)

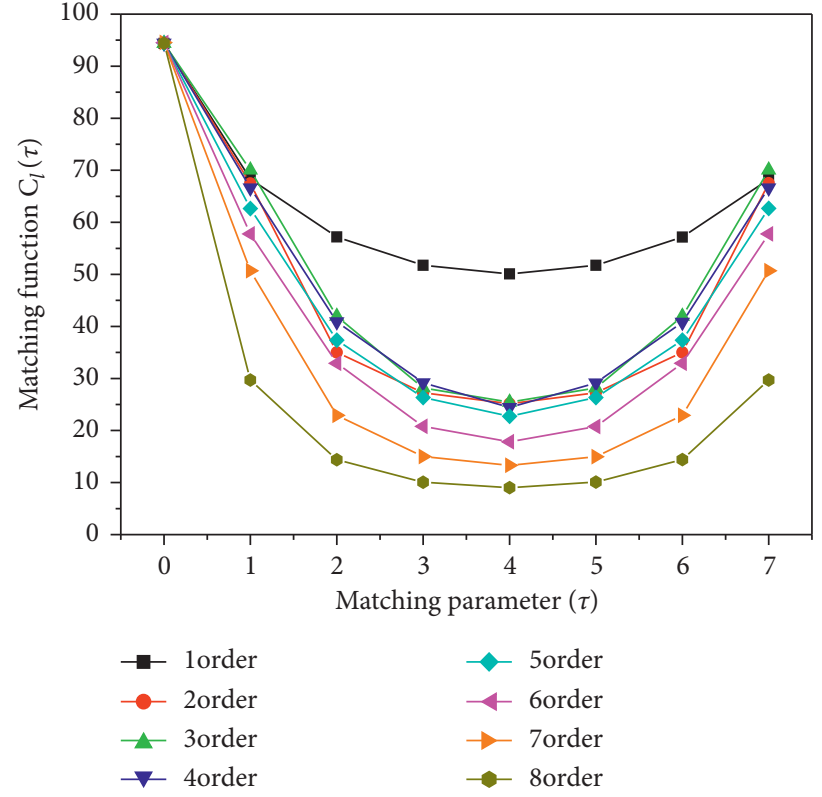

(b)

Figure 5: Vibration mode matching function of weakly coupling mistuned bladed disk. (a) Mobile interface method. (b) Fixed-interface method.
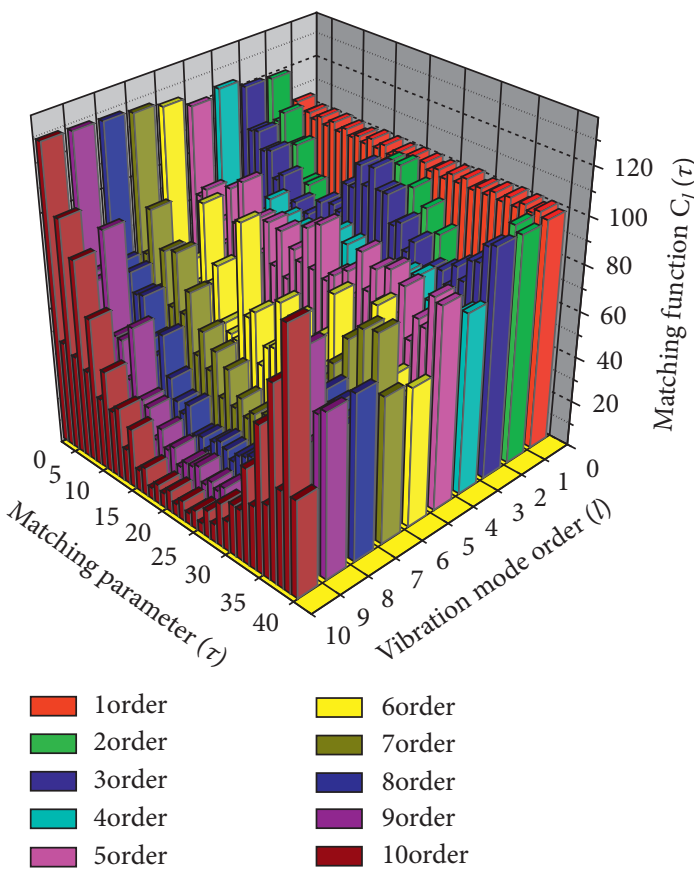

(a)

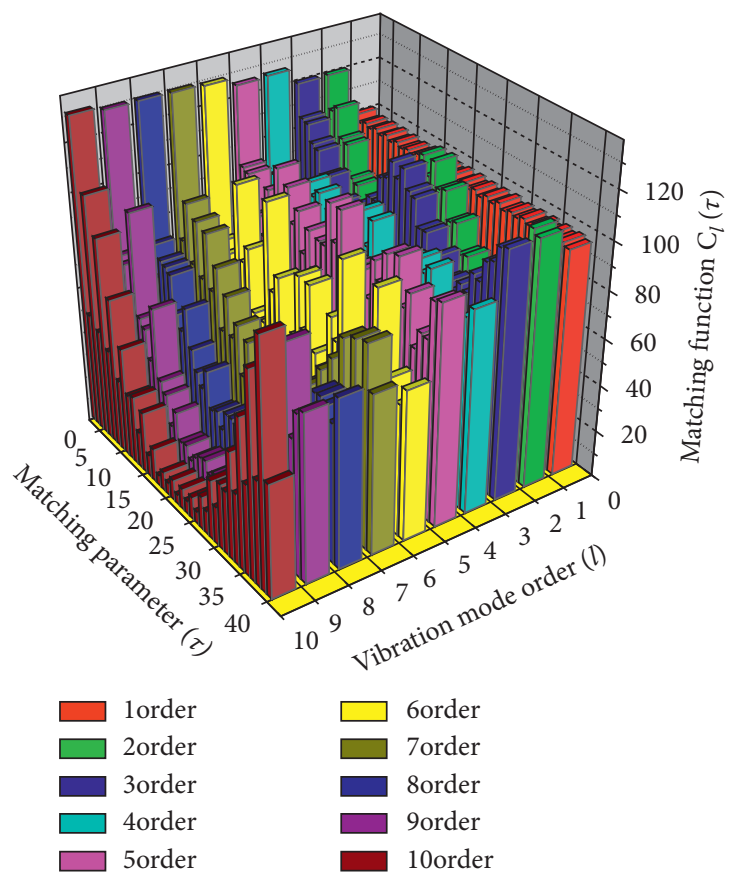

(b)

FIGURE 6: Vibration mode matching function of generally coupling mistuned bladed disk. (a) Mobile interface method. (b) Fixed-interface method. 


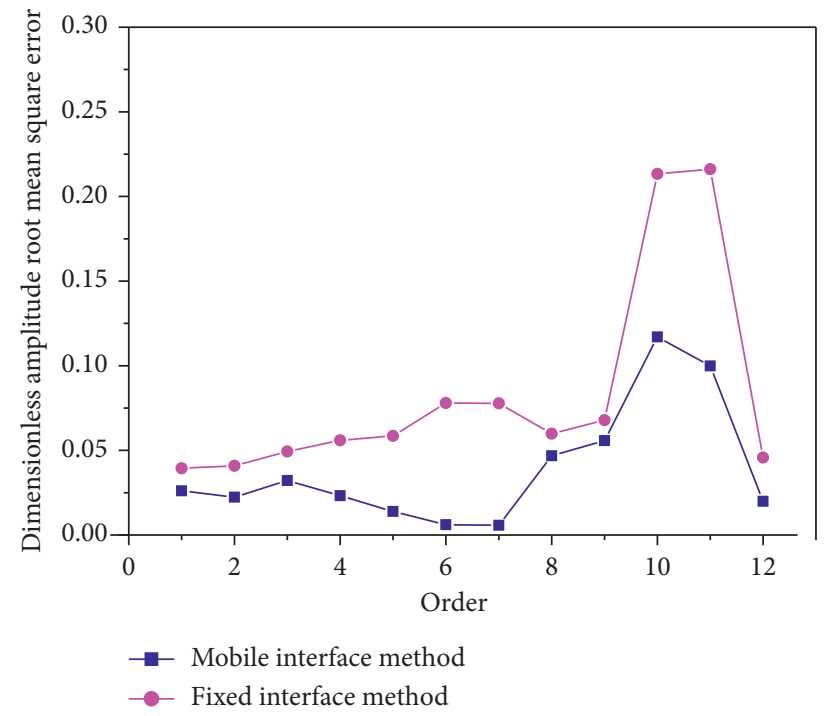

FIgURE 7: Root mean square error of dimensionless vibration amplitude of strongly coupling mistuned bladed disk.

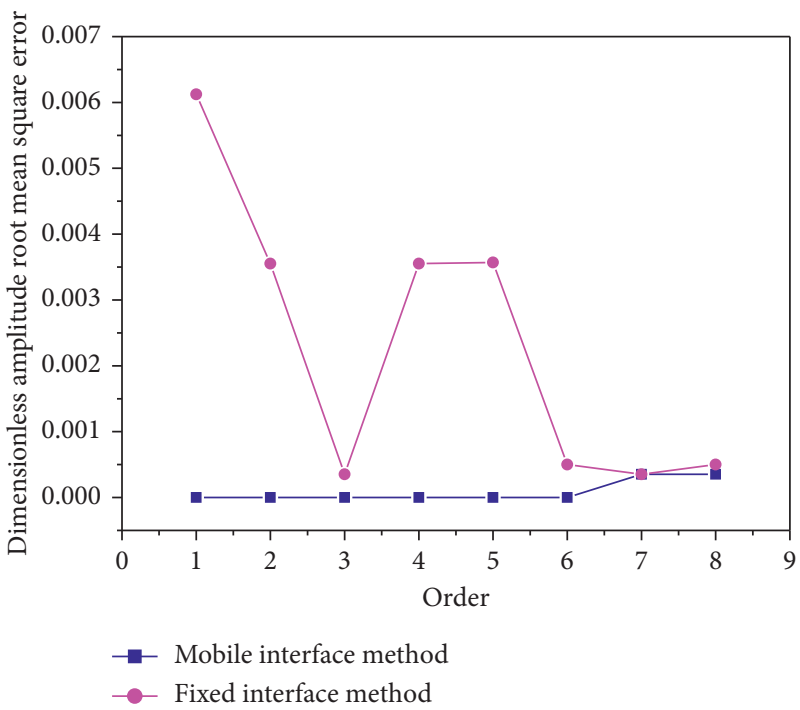

FIGURE 8: Root mean square error of dimensionless vibration amplitude of weakly coupling mistuned bladed disk.

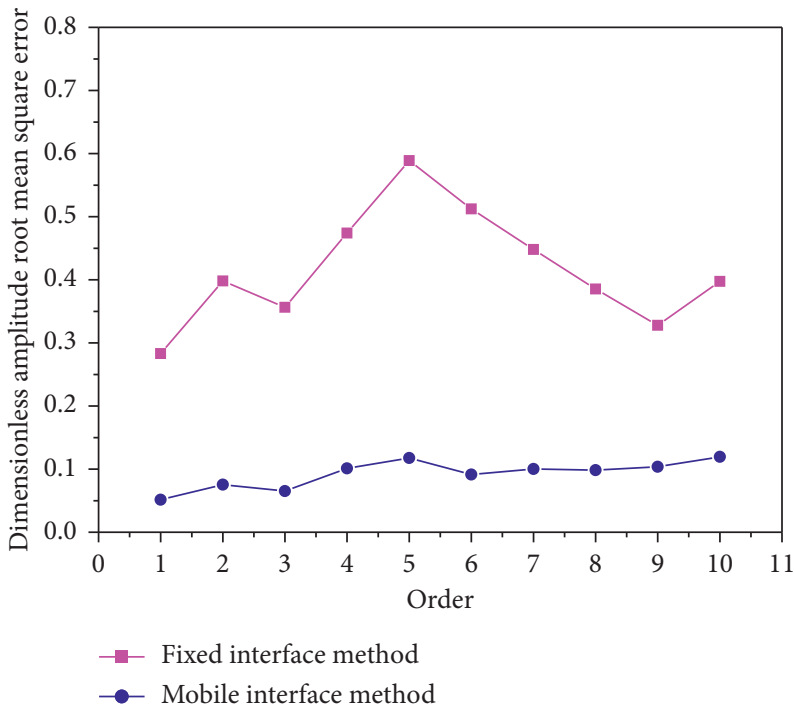

FIgURE 9: Root mean square error of dimensionless vibration amplitude of generally coupling mistuned bladed disk. 
TABLE 3: The analysis accuracy of dynamic frequencies and analysis time of three methods.

\begin{tabular}{|c|c|c|}
\hline Analysis method & Analysis accuracy of dynamic frequencies & Analysis time \\
\hline Mobile interface prestressed CMS superelement method & $\begin{array}{c}\text { Strong coupling: } 99.8534 \% \\
\text { General coupling: } 99.9039 \% \\
\text { Weak coupling: } 100 \%\end{array}$ & General coupling: $28.42 \%$ \\
\hline Fixed-interface prestressed CMS superelement method & $\begin{array}{l}\text { Strong coupling: } 70.1860 \% \\
\text { General coupling: } 97.1675 \% \\
\text { Weak coupling: } 100 \%\end{array}$ & General coupling: $27.07 \%$ \\
\hline Direct FEM method & $100 \%$ & General coupling: $100 \%$ \\
\hline
\end{tabular}

basically the same as that of the fixed-interface method, but the analysis accuracy is better than that of the fixed-interface method, especially for strongly coupling bladed disk.

\section{Conclusions}

Aiming at the shortcoming of the existing methods for predicting the vibration characteristics of bladed disk structures and other large and complex structures, a mobile interface prestressed CMS superelement method is proposed in this paper. Taking the prediction results of the direct method as the theoretical value, the relative error of dynamic frequency, the mode matching function, and the dimensionless root mean square error of vibration amplitude verify the prediction accuracy of the proposed method. The prediction accuracy of the proposed method is compared with that of the fixed-interface CMS method. The specific conclusions are as follows:

(1) For the strongly coupled mistuned bladed disk structure, the relative error of dynamic frequency and the root mean square error of dimensionless amplitude predicted by the mobile interface method are small and the mode patterns are matched. The dynamic frequency relative error and the root mean square error of the dimensionless amplitude predicted by the fixed-interface method are large and the mode mismatch occurs.

(2) For the weakly coupled mistuned bladed disk structures, the relative errors of dynamic frequencies predicted by the mobile interface method and fixedinterface method are both $0 \%$, and the modes are matched, but the dimensionless root mean square error of amplitude predicted by mobile interface method is less than that by the fixed-interface method.

(3) For the general coupled detuned bladed disk structures, the relative error of dynamic frequency predicted by the mobile interface method is small and basically unchanged, while the dynamic frequency relative error predicted by the fixed-interface method is large when the order is low, and the relative error of dynamic frequency decreases gradually with the increase of order. The mode shapes predicted by the two methods match those of the direct method, while the root mean square error of the dimensionless amplitude predicted by the mobile interface method is less than that by the fixed-interface method.
(4) The mobile interface method is more accurate in predicting the vibration characteristics of the mistuned bladed disk structures with different coupling degrees. Compared with the direct FEM method, this method is less time-consuming.

\section{Data Availability}

The data used to support the findings of this paper are available from the corresponding author upon request.

\section{Conflicts of Interest}

The authors declare that there are no conflicts of interest regarding the publication of this paper.

\section{Acknowledgments}

This article was supported by the National Natural Science Foundation of China (no. 51505206), Liaoning Provincial Natural Science Foundation Guidance Program Project (no. 2019-ZD-0694), Liaoning Provincial Department of Education Scientific Research General Project (no. L2014246), and Liaoning University of Technology Teacher Research Start Fund Project (no. X201202). The authors are grateful to them.

\section{References}

[1] C. Y. Zhou, J. X. Zou, X. Y. Wen, and H. Y. Sheng, "Coupled vibrations of gas turbine shrouded blades," Journal of Harbin Institute of Technology, vol. 33, no. 1, pp. 129-133, 2001.

[2] H. Q. Yuan, L. Zhang, and Q. K. Han, "Optimization of mistuning blades an aero-engine based on arrangement for vibration absorption in artificial ant colony algorithm," Journal of Vibration and Shock, vol. 31, no. 11, pp. 169-172, 2012.

[3] J. J. Wang, C. B. Yu, and Q. H. Li, "Localization characteristics of vibratory mode for bladed disk assemblies," Journal of Aerospace Power, vol. 24, no. 4, pp. 788-792, 2009.

[4] F. Qin and L. M. Chen, "Vibration analysis of mistuned bladed disc of steam turbine," Journal of Beijing University of Technology, vol. 33, no. 2, pp. 126-128, 2007.

[5] I. Lee, S. Shin, and Y. Kim, "Mistuned bladed disk forced vibration analysis based on standing wave formulation," Aerospace Science and Technology, vol. 24, no. 1, pp. 275-282, 2013.

[6] X. Kan, Z. Xu, B. Zhao, and J. Zhong, "Effect of coriolis force on forced response magnification of intentionally mistuned 
bladed disk," Journal of Sound and Vibration, vol. 399, pp. 124-136, 2017.

[7] J. Yuan, F. Scarpa, G. Allegri, B. Titurus, S. Patsias, and R. Rajasekaran, "Efficient computational techniques for mistuning analysis of bladed discs: a review," Mechanical Systems and Signal Processing, vol. 87, pp. 71-90, 2017.

[8] R. J. Guyan, "Reduction of stiffness and mass matrices," AIAA Journal, vol. 3, no. 2, p. 380, 1965.

[9] B. Irons, "Structural eigenvalue problems-elimination of unwanted variables," AIAA Journal, vol. 3, no. 5, pp. 961-962, 1965.

[10] M. Paz, "Dynamic condensation," AIAA Journal, vol. 22, no. 5 , pp. $724-727,1984$.

[11] W. C. Hurty, "Vibrations of structural systems by component mode synthesis," Journal of the Engineering Mechanics Division, vol. 86, no. 4, pp. 51-69, 1960.

[12] S. Hou, "Review of mode synthesis techniques and a new approach," Shock and Vibration Bulletin, vol. 40, no. 4, pp. 25-30, 1969.

[13] R. L. Goldman, "Vibration analysis by dynamic partitioning," AIAA Journal, vol. 7, no. 6, pp. 1152-1154, 1969.

[14] S.-T. Wei and C. Pierre, "Statistical analysis of the forced response of mistuned cyclic assemblies," AIAA Journal, vol. 28, no. 5, pp. 861-868, 1990.

[15] P. Vargiu, C. M. Firrone, S. Zucca, and M. M. Gola, "A reduced order model based on sector mistuning for the dynamic analysis of mistuned bladed disks," International Journal of Mechanical Sciences, vol. 53, no. 8, pp. 639-646, 2011.

[16] C. Y. Zhou, J. X. Zou, X. Y. Wen, and H. Y. Shen, "Vibration characteristics analysis of mistuned blade-disc systems of gas turbine," Gas Turbine Technology, vol. 13, no. 3, pp. 42-46, 2000.

[17] P. Y. Wang and L. Li, "Reduced order computational method for analysis of mistuning bladed disk dynamics characteristic," Journal of Aerospace Power, vol. 29, no. 6, pp. 1395-1402, 2014.

[18] C. P. Zang, Y. L. Duan, and E. P. Petrov, "Reduced-order modelling and dynamic response prediction method for mistuned bladed disks," Acta Aeronautica et Astronautica Sinica, vol. 36, no. 10, pp. 3305-3315, 2015.

[19] L. Zhang, Research on Vibration and Multi-Field Coupling Mechanics Characteristic of Bladed Disk System in Aero-Engine, Northeastern University, Shenyang, china, 2013.

[20] L. Zhang and X. Li, "Study on dynamic characteristics of mistuned bladed disk system based on approximate CMS method and modal testing," China Measurement \& Test, vol. 42, no. 6, pp. 117-121, 2016.

[21] Z. Li, W. Yang, and H. Yuan, "Vibration analysis of aeroengine blisk structure based on a prestressed CMS superelement method," Shock and Vibration, vol. 2016, Article ID 1021402, 10 pages, 2016.

[22] H. Y. Zhang, H. Q. Yuan, W. J. Yang, and T. Y. Zhao, "Characteristics of frequency veering for mistuned bladed disks based on the contribution degree of bladed," Journal of Vibration, Measurement \& Diagnosis, vol. 39, no. 2, pp. 106-111+217, 2019.

[23] H. Y. Zhang, H. Q. Yuan, W. J. Yang, and T. Y. Zhao, "Optimization of vibration reduction of mistuned blades based on pre-tressed component mode synthesis method," Journal of South China University of Technology, vol. 46, no. 2, pp. 79-86, 2018.

[24] H. X. Li, H. Q. Yuan, and L. X. Zhang, "Study on key factors of mistuned vibration for blade-disk system of some-stage compressor," Journal of Aerospace Power, vol. 32, no. 5, pp. 1082-1090, 2017.
[25] H. Y. Sun, H. Q. Yuan, and T. Y. Zhao, "Study on vibration characteristics of mistuned blisk based on PIHISCMSM," Journal of Northeastern University, vol. 41, no. 12, pp. 1733-1740, 2020.

[26] L. Zhang, Y. He, H. Yuan, and X. Li, "Vibration characteristics analysis of mistuned bladed disk system based on mobile interface prestressed CMS super-element method," Journal of Vibroengineering, vol. 20, no. 7, pp. 2576-2592, 2018. 